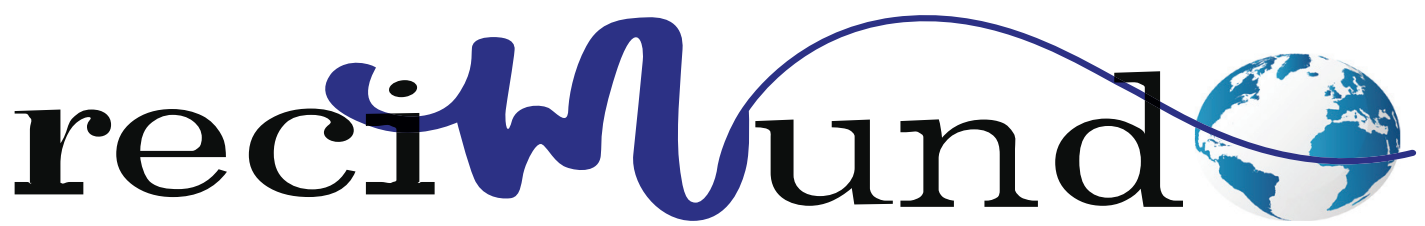

Revista Científica Mundo de la Investigación y el Conocimiento

DOI: 10.26820/recimundo/4.(1).esp.marzo.2020.143-154

URL: http://recimundo.com/index.php/es/article/view/785

EDITORIAL: Saberes del Conocimiento

REVISTA: RECIMUNDO

ISSN: 2588-073X

TIPO DE INVESTIGACIÓN: Artículo de Revisión

CóDIGO UNESCO: 3205 Medicina Interna

PAGINAS: $143-154$

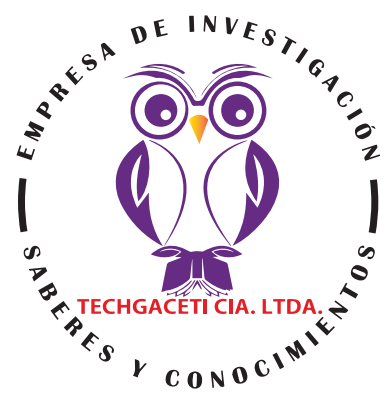

\title{
Lumbago: rizotomia facetaria
}

Lumbago: facet rhizotomy

Lumbago: rizotomia faceta

Elizabeth Johanna Pillco Guaman'; Monica María Pañega Narváez;

Roberta del Carmen López Aldas ${ }^{3}$; Christian Fernando Cusco Rivera ${ }^{4}$

\section{RECIBIDO: 20/11/2019 ACEPTADO: 29/01/2020 PUBLICADO: 05/03/2020}

1. Médico; Químico Farmacéutica; Investigadora Independiente; Cuenca, Ecuador; joiseliza@hotmail.com; (D) https://orcid.org/0000-0002-7220-5529

2. Médico; Investigadora Independiente; Cuenca, Ecuador; monikmerydey@gmail.com; (D) https://orcid.org/00000002-8436-2441

3. Médico General; Investigadora Independiente; Riobamba, Ecuador; robertalopa_91@hotmail.com; (DD https:// orcid.org/0000-0002-9707-4983

4. Médico; Investigador Independiente; Cuenca, Ecuador; cris_cuzco@hotmail.com; (D) https://orcid.org/00000002-6971-2482

CORRESPONDENCIA

Elizabeth Johanna Pillco Guaman

joiseliza@hotmail.com

Cuenca, Ecuador

( RECIMUNDO; Editorial Saberes del Conocimiento, 2020 


\section{RESUMEN}

El lumbago, termino corriente con el que se conoce la lumbalgia, está referido al dolor en la columna lumbar (zona baja de la espalda), es decir, el área ubicado entre las últimas costillas y la zona glútea. Es una condición que, en gran medida, aflige a la población a nivel mundial y desde hace más de 25 años se ha observado un incremento exponencial en este tipo de complicaciones. La condición del síndrome facetario lumbar se ubica entre las etiologías estructurales de dolor de espalda y su causa es la degeneración de las articulaciones facetarias, las cuales sufren los cambios propios de cualquier articulación sinovial. El manejo fundamental del mismo ha sido representado más recientemente por tratamientos consistentes en bloqueos nerviosos y rizolisis o neurolisis de la rama medial del ramo dorsal del nervio espinal. La rizotomía es especialmente considerada la técnica mínimamente invasiva más usada en el manejo del dolor facetario lumbar, por su baja tasa de complicaciones $(<1 \%)$, su practicidad en la ejecución y bajos costos asociados. El enfoque del presente estudio es efectuar una investigación documental, mediante una metodología de revisión que facilite efectuar una aproximación al tema del tratamiento de la lumbalgia de origen facetario mediante la técnica de la rizotomía. Los resultados sirvieron para aportar algunas concepciones recientes en cuanto al lumbago, así como también sus factores de riesgo. Paralelamente se desarrolla lo relativo a síndrome facetario, diagnóstico y rizotomía facetaria. Se concluye destacando que, en base a la literatura encontrada fue posible inferir que aún hay razonados estudios que demuestran que dicha técnica es considerada el tratamiento de elección idóneo para el tratamiento de la lumbalgia, sobretodo, tras obtener resultados insatisfactorios con las terapias conservadoras.

Palabras clave: Rizólisis, Denervación, Diatermocoagulación, Neuroablación, Articulación Zigoapofisaria

\section{ABSTRACT}

Lumbago, a common term with which low back pain is known, refers to pain in the lumbar spine (lower back area), that is, the area between the last ribs and the gluteal area. It is a condition that, to a large extent, afflicts the population worldwide and for more than 25 years an exponential increase in this type of complications has been observed. The condition of the lumbar facet syndrome is among the structural etiologies of back pain and its cause is the degeneration of the facet joints, which undergo the changes of any synovial joint. Its fundamental management has been represented more recently by treatments consisting of nerve blocks and rhizolysis or neurolysis of the medial branch of the dorsal branch of the spinal nerve. Rhizotomy is especially considered the minimally invasive technique most commonly used in the management of lumbar facet pain, due to its low complication rate $(<1 \%)$, its practicality in execution and low associated costs. The focus of the present study is to carry out a documentary investigation, through a review methodology that facilitates an approach to the topic of treatment of low back pain of facet origin by means of the rhizotomy technique. The results served to provide some recent conceptions regarding lumbago, as well as its risk factors. In parallel, the aspects related to facet syndrome, diagnosis and facet rhizotomy are developed. It is concluded by highlighting that, based on the literature found, it was possible to infer that there are still reasoned studies that show that this technique is considered the ideal treatment for the treatment of low back pain, especially after obtaining unsatisfactory results with conservative therapies.

Keywords: Rhizolysis, Denervation, Diathermocoagulation, Neuroablation, Zygoapophysial Joint

\section{RESUMO}

Lumbago, um termo comum com o qual a dor lombar é conhecida, refere-se à dor na coluna lombar (região lombar), ou seja, a área entre as últimas costelas e a área glútea. É uma condição que, em grande medida, afeta a população em todo o mundo e há mais de 25 anos observa-se um aumento exponencial desse tipo de complicação. A condição da síndrome da faceta lombar está entre as etiologias estruturais da dor nas costas e sua causa é a degeneração das articulações da faceta, que sofrem as alterações de qualquer articulação sinovial. Seu manejo fundamental foi representado mais recentemente por tratamentos que consistem em bloqueios nervosos e rizólise ou neurólise do ramo medial do ramo dorsal do nervo espinhal. A rizotomia é especialmente considerada a técnica minimamente invasiva mais utilizada no tratamento da dor nas facetas lombares, devido à baixa taxa de complicações (<1\%), praticidade na execução e baixos custos associados. O foco do presente estudo é realizar uma investigação documental, através de uma metodologia de revisão que facilite a abordagem do tópico tratamento da lombalgia de origem facetária por meio da técnica da rizotomia. Os resultados serviram para fornecer algumas concepções recentes sobre lumbago, bem como seus fatores de risco. Paralelamente, são desenvolvidos os aspectos relacionados à síndrome facetária, diagnóstico e rizotomia facetária. Conclui-se destacando que, com base na literatura encontrada, foi possível inferir que ainda existem estudos fundamentados que mostram que essa técnica é considerada o tratamento ideal para o tratamento da lombalgia, principalmente após a obtenção de resultados insatisfatórios com terapias conservadoras.

Palavras-chave: Rizólise, Denervação, Termocoagulação Dia, Neuroablação, Articulação Zigapofisária. 


\section{Introducción}

El dolor de espalda, según las fuentes referidas por Martínez et al. (2017), y más específicamente el relacionado con la columna lumbar, es una condición que, en gran medida, aflige a la población a nivel mundial; incluso, el 25\% de la misma llega a padecer al menos un episodio de dolor lumbar anualmente, por lo que dicha situación alcanza a representar un impacto socioeconómico importante, a la par de considerables porcentajes de ausentismo y bajas laborales.

No obstante, Minda, Saavedra, García, \& López (2020), refirieron estudios de menos de diez años en los que afirmó que, desde hace más de 25 años se ha observado un incremento exponencial en este tipo de complicaciones, y adicionalmente proyectaban que:

El 90\% de la población general tendrá dolor lumbar a lo largo de sus vidas, estimándose aproximadamente que $5 \%$ de ellos lo tendrá todos los años en sus distintas formas y solo $1 \%$ corresponderían al dolor crónico e incapacitante. (Párr. 5)

La lumbalgia, según Fundación Española de Reumatología (s.f.) es coloquialmente conocida como "lumbago" esté referido al dolor en la columna lumbar (zona baja de la espalda), es decir, el área ubicado entre las últimas costillas y la zona glútea, el cual es originado por:

alteraciones de las diferentes estructuras que forman la columna vertebral a ese nivel, como ligamentos, músculos, discos vertebrales y vértebras. Aproximadamente, el $85 \%$ de la población va a sufrir este tipo de dolor en algún momento de su vida, siendo la inmensa mayoría de las veces de causa benigna. Hablamos de lumbalgia aguda si dura menos de 6 semanas, y lumbalgia crónica cuando la duración del dolor es superior a este periodo.

Por su parte, Jarufe (s.f.) como experto en el área aclara que, si bien pudiera no pa- decerse de hernias, compresiones o de cualquier enfermedad propia de algún tipo de intervención quirúrgica, ello tampoco implica que sea una condición suficiente para que una persona cualquiera quede exenta de dolores lumbares o de otros segmentos de la columna. De hecho, detalla que:

Múltiples factores determinan la aparición de dolores en la columna. Entre ellos, tal vez los más frecuentes son los fenómenos asociados a la sobrecarga de las articulaciones que unen lateralmente las vértebras en sentido superior-inferior. Estas articulaciones se llaman articulaciones facetarias por estar formadas por un complejo dependiente de las facetas articulares de las vértebras. Se trata de pequeñas articulaciones que sufren con el tiempo procesos degenerativos normales, acelerados y agravados por factores dependientes de cada individuo. Entonces se llegará en algún momento de la vida, en general después de los 30 años y principalmente después de los 40, a la presencia de artrosis facetaria o espóndil-artrosis en estos segmentos, donde el más afectado es el segmento de la columna lumbar. La artrosis lleva a un aumento reactivo del tamaño de las facetas y puede producirse dolor por afectación de los nervios que dan la sensibilidad a estas articulaciones. Cuando se producen síntomas debido a estos cambios (lumbago y otros, como dolores cervicales), se llega al llamado síndrome facetario. (Párr. 2)

En este mismo orden de ideas, Martínez et al. (2017) también precisa que, es dentro de las etiologías estructurales de dolor de espalda que se ubica la condición del síndrome facetario lumbar, y en relación a ello destaca que:

Su prevalencia como causa de dolor lumbar está entre el 15-31\% con incidencia a lo largo de la vida de hasta el 52\% en algunas series. Su causa es la degeneración de las articulaciones facetarias, las cuales sufren los cambios propios de cualquier articulación sinovial. Clínicamente cursa con dolor 
lumbar de características mecánicas que aumenta con los movimientos de rotación y flexo-extensión (en especial en hiperextensión). No es rara su irradiación a nalgas y cara posterior de muslos. El comienzo y el curso suelen ser insidiosos, aunque muchos pacientes lo refieren a raíz de un esfuerzo brusco. [...]

Actualmente el abordaje estándar de tratamiento consiste en bloqueos nerviosos y rizolisis o neurolisis de la rama medial del ramo dorsal del nervio espinal. (págs. 7-8)

Minda, Saavedra, García, \& López (2020) de acuerdo a sus fuentes afirma que "La rizotomía es el procedimiento no invasivo más utilizado para el manejo del dolor facetario lumbar, debido a su baja tasa de complicaciones $(<1 \%)$, su facilidad de aplicación y los bajos costos asociados"

Esta terapia, que también puede ser conocida como: risolisis (rizotomía) por radiofrecuencia, denervación (desnervación) por radiofrecuencia, diatermocoagulación, ablación por radiofrecuencia, coagulación térmica, entre otras; según Meli (2016) es efectiva contra el dolor de columna causado por la inflamación de la articulación de las facetas vertebrales (también conocida como articulación intervertebral, articulación zigoapofisaria o carillas articulares). Estas estructuras son extremadamente sensibles a los procesos inflamatorios y a los cambios por artrosis propios de la edad.

En definitiva, la literatura de manera general hace referencia a que esta técnica neuroablativa bien puede aplicar para el tratamiento del dolor lumbar, cervical y/o dorsal, es decir, lumbalgia, cervicalgia y dorsalgia, respectivamente. Concebido como una intervención mínimamente invasiva y sencilla, que es posible llevar a cabo de manera ambulatoria, mediante anestesia local y con apoyo radiológico o ecográfico, la cual es especialmente indicada para aquellos casos de pacientes intervenidos por un equipo médico multidisciplinar con terapias conservadoras que no terminaron siendo efectivas, recidivando sus trastornos dorsales por largo tiempo; principales razones por las que terminan considerando el dolor de espalda con causas originadas probablemente en los nervios colindantes a la columna vertebral. Adicionalmente también se encentra que dicha práctica es utilizada para aliviar el dolor bien focalizado y no irradiado hacia otras zonas del cuerpo.

Coincidiendo con la versatilidad de dicha intervención, Madariaga (2020) agrega que también es normalmente recomendada desde las Unidades del Dolor en pro de mejora a mediano y largo plazo del dolor que se origina en las facetas o articulaciones zigoapofisarias, precisando que el mismo es notable por el paciente especialmente al comenzar cualquier actividad después de un periodo de reposo, el cual paulatinamente se reduce tras sostener una actividad moderada.

El enfoque del presente estudio es efectuar una investigación documental, mediante una metodología de revisión que facilite efectuar una aproximación al tema del tratamiento de la lumbalgia de origen facetario mediante la técnica de la rizotomía, por lo que, como resultado se presentaran, algunas acepciones fundamentales que expliquen el lumbago (lumbalgia) como patología, para luego terminar con el referenciación de otras fuentes que aborden su tratamiento mediante el mencionado proceso.

\section{Materiales y Métodos}

La presente investigación se ha concebido en base a un diseño bibliográfico, a su vez aplicando una metodología de revisión puesto que, como se ha dicho, el objeto es realizar un acercamiento teórico sobre el aspecto terapéutico de la rizotomía en el lumbago.

Fue a finales de febrero del corriente que se inició una búsqueda de literatura, tanto en bases de datos como, por ejemplo: MedlinePlus, BVS, Embase, Medigraphic, 
Researchgate, Infomed, SciELO, Scopus Elsevier, entre otras; así como también en otros sitios webs de organizaciones, asociaciones, instituciones, fundaciones y sociedades de carácter nacional, regional e internacional, enfocadas en el área de la salud, medicina, neurología, neurocirugía y traumatología.

Cada una de las pesquisas se hicieron mediante el uso de expresiones útiles como criterios de preselección, destacándose entre estas: Lumbago, Rizotomía Facetaria, Lumbalgia, Rizólisis Facetaria y Denervación/Denervación por radiofrecuencia; con las que se obtuvo una variedad de resultados que redireccionaban a distintos tipos de fuentes bibliográficas.

Necesariamente, estas derivaciones se fueron refinando en razón de: correlación y relevancia temática, preferiblemente publicados en idioma español o inglés en el periodo de los últimos diez años, escogiendo con prioridad aquella literatura relativa a: artículos científicos (con y sin metadatos), protocolos, consensos, manuales de procedimiento, guías clínicas, boletines informativos, ensayos, tesis de grado, posgrado o doctorado, informes y proyectos, directrices, folletos, resúmenes, libros digitalizados (o electrónicos) y cualquier otro tipo de información o recurso bibliográfico en formato digital proveniente de los mencionados canales de informativos, accesible completa y gratuitamente.

Los recursos bibliográficos desestimados estuvieron representados por: editoriales, cartas al editor, anotaciones académicas y otros contenidos considerados de escaso valor cientificoacadémico por su nulo o muy bajo nivel de evidencia referido.

Todo este proceso derivó en el correspondiente análisis crítico y discusión entre los miembros del equipo investigador, entendiéndose por esto, el desarrollo de lecturas individuales y grupales que, en definitiva, sirvieron para, consensuadamente, compilar y plasmar el contenido expuesto en la presente entrega. Todos estos procesos, en general, constituyeron fundamentalmente las fases de la presente investigación, que además sirvió para una mayor comprensión del tema y facilitó la síntesis de la mejor evidencia disponible, lo que, por cierto, significa uno de los resultados esperados.

\section{Resultados}

\section{Lumbago (Lumbalgia)}

El término lumbalgía o lumbago se define como todo aquel dolor común de duración variable, circunscrito a la parte baja de la espalda o zona lumbar. Éste suele ser intenso y profundo, teniendo como efecto final una repercusión en la movilidad normal de la zona, debido a la sensación dolorosa. (Aguilera \& Herrera, 2013)

De conformidad con la Agencia para la Investigación y la Calidad de la Atención Médica (AHRQ por su sigla en inglés) el lumbago pudiera asociarse con:

...un dolor no agudo y fastidioso o un dolor agudo y punzante. El dolor puede ser leve o intenso y se puede sentir hormigueo o ardor. También puede haber dolor en las piernas. En su forma aguda, el lumbago dura 4 semanas o menos. En la forma subaguda, dura entre 4 y 12 semanas. El lumbago crónico dura 12 semanas o más. (AHRQ, 2017)

En la Enciclopedia Ilustrada de Salud de A.D.A.M. (2018) (2019) se distingue entre: Lumbago Agudo, cuando el dolor lumbar se extiende en un periodo de tiempo que puede comprender algunos días e incluso pocas semanas; y el Lumbago-Crónico, relacionado con aquel que se prolonga en el tiempo.

\section{Factores de Riesgo del Lumbago (Lum- balgia)}

El Instituto Nacional de Trastornos Neurológicos y Accidentes Cerebrovasculares

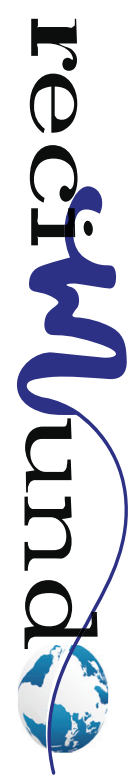


(NINDS, por sus siglas en ingles) de los Estados Unidos de Norteamérica afirma que, hay factores determinados que pueden elevar la posibilidad de padecer dolor lumbar, independientemente de la existencia de enfermedades subyacentes, y entre estos están:

- La Edad: el primer ataque de dolor en la parte baja de la espalda ocurre por lo general entre los 30 y 50 años de edad, y el dolor se vuelve más común con la edad. [...]

- El estado físico: el dolor lumbar es más común entre las personas que no están en buen estado físico. Si los músculos de la espalda y el abdomen están debilitados, es posible que no puedan soportar correctamente la columna vertebral. [...]

- El embarazo: viene comúnmente acompañado de dolor en la parte baja de la espalda como resultado de los cambios en la pelvis y el aumento de peso. Estos síntomas casi siempre desaparecen después del parto. [...]

- El eamento de peso: el sobrepeso, la obesidad o aumentar rápidamente una cantidad significativa de peso puede poner mayor presión sobre la espalda y causar dolor lumbar.

- Factores genéticos: algunas causas de dolor lumbar, tales como la espondilitis anquilosante, una forma de artritis que involucra la fusión de las articulaciones de la columna y causa alguna inmovilidad de la columna vertebral, tienen un componente genético.

- Factores de riesgo ocupacionales: el tener un trabajo que requiera levantar, empujar o halar cosas pesadas, especialmente cuando involucra torcedura o vibración de la columna, puede causar lesiones y dolor lumbar. Un trabajo inactivo o un trabajo de escritorio también puede causar o contribuir al dolor, espe- cialmente si la persona tiene una mala postura o se sienta todo el día en una silla sin suficiente soporte lumbar.

- Factores de salud mental: los problemas de salud mental preexistentes, tales como la ansiedad y la depresión, pueden influir en cómo o cuánto la persona se enfoca en su dolor, así como en su percepción de la gravedad del mismo. El dolor que se vuelve crónico también puede contribuir al desarrollo de dichos factores psicológicos. El estrés puede afectar al cuerpo de muchas maneras, incluso causando tensión muscular.

- Sobrepeso de las mochilas que llevan los niños: el dolor lumbar no relacionado con lesión u otra causa conocida es poco común en los niños preadolescentes. Sin embargo, una mochila sobrecargada de libros y materiales de la escuela puede poner mucha tensión sobre la espalda y causar fatiga muscular. La Academia Americana de Cirujanos Ortopédicos recomienda que la mochila de un niño no deba pesar más de 15 a 20 por ciento del peso corporal del niño. (NINDS, 2016)

Ahora bien, en base a que el tratamiento al que se hace referencia en esta investigación se encuentra asociado a una sintomatología determinada; aspecto que es aclarado por Aguilera \& Herrera (2013) al referir de conformidad a sus fuentes que "la lumbalgia no es una enfermedad, sino más bien un síntoma que puede ocurrir por distintas razones" (Pág. 80), se debe comprender entonces que, al hacer referencia a la rizotomía facetaria, es porque esta naturalmente se encuentra vinculada a un tratamiento para el dolor generado en los segmentos de la columna conocidos como facetas articulares, que en ningún caso guarda relación con hernias discales, ni estrechamientos, ni advierte un compromiso neurológico. es decir, se trata de la condición que, en términos generales, se vincula con el conocido síndrome facetario. 


\section{Síndrome Facetario}

Es un conjunto de síntomas y signos producidos principalmente por fenómenos patológicos de las articulaciones facetarias en los diferentes segmentos de la columna, principalmente en la columna lumbar. Esto lleva a desarrollar lumbagos mecánicos crónicos, con ausencia en general de componentes como hernias de los discos y estrechamientos significativos del canal. (Jarufe, s.f.)

Rodríguez (2020) explica que las articulaciones facetarias son como las articulaciones existentes en las rodillas o los hombros, pero en este caso se refieren a las conexiones entre las vértebras en la columna, que admiten los movimientos de inclinación o torcimiento y además contienen los nervios que salen de estas mediante los cuales se transmiten los impulsos dolorosos al cerebro. El efecto de una lesión e incluso la artritis, puede ser la causa de un proceso inflamatorio en estas, a su vez generando dolor (en algunos casos irradiantes hacia la zona del glúteo y región fémur femoral, y por lo general sin extenderse más allá de la rodilla) e inflexibilidad. Entonces, es así como el síndrome de la articulación facetaria es el que con frecuencia causa la lumbalgia (lumbago) u otros dolores asociados con la columna, y es posible confirmar su diagnóstico cuando se consigue anular el dolor tras inyectar (infiltrar) anestesia local y un antiinflamatorio en las articulaciones afectadas.

De la misma manera, el tratadista aclara que, es en los casos crónicos de este, cuando las inyecciones producen un corto alivio del dolor, que se recomienda la rizotomía por radiofrecuencia; procedimiento por medio del cual puede bloquearse la transmisión de dolor al someter dichos nervios a un calentamiento con ondas de radiofrecuencia. Con este método, generalmente, es posible conseguir aliviar el dolor por períodos que oscilan entre los 6 y 24 meses.

\section{Diagnóstico del Síndrome Facetario}

Martínez et al. (2017) recientemente alega- ron que, para el diagnóstico del síndrome facetario lumbar el estándar es el bloqueo de la rama medial del ramo dorsal del nervio espinal, misma que asiste la inervación sensitiva a las articulaciones interapofisarias. De hecho, insisten en que este es el medio idóneo e igualmente necesario que, por la evidencia disponible, sin lugar a dudas se realiza en los casos del diagnóstico de síndrome facetario previo a las técnicas neuroablativas. (pág. 7)

La Asociación Internacional para el estudio del Dolor (AIED) recomienda que el dolor facetario se diagnostique sólo con base en inyecciones articulares controladas radiológicamente (utilizando un placebo) o comparativas (dos anestésicos locales diferentes) o bloqueos de rama medial. Factores tales como el riesgo relativo de otro bloqueo falso positivo, la tasa de complicaciones del segundo bloqueo de diagnóstico versus el procedimiento definitivo (denervación), los falsos negativos y la rentabilidad dictan la práctica clínica. Además, estos bloqueos, solos, forman parte del armamento terapéutico. [...]

El diagnóstico de dolor facetario es clínico y no radiográfico. El grado de degeneración identificado en la evaluación radiológica no se correlaciona con la gravedad del dolor en las articulaciones facetarias. Sin embargo, si el paciente tiene una resonancia magnética normal (RMN) o tomografía computarizada (TC) de la columna vertebral es poco probable que su dolor sea en articulación de la faceta. Hay dos maneras de realizar un bloqueo de diagnóstico: (1) se inyecta con un anestésico local en la propia articulación o (2) anestesiar las ramas mediales de las raíces nerviosas espinales que inervan una articulación facetaria particular.

Si la articulación facetaria es el generador de dolor, tanto el bloqueo intra-articular como el bloqueo medial de la rama aliviarán temporalmente el dolor producido por la articulación facetaria. Normalmente, los pacientes reciben un diario de dolor antes 
de la inyección e inmediatamente después del procedimiento. El bloqueo se considera positivo si alivia el dolor en la zona de la que se podría esperar que el dolor se bloquee. Si el dolor no se alivia, una nueva hipótesis sobre la fuente del dolor del paciente necesita ser desarrollada. (Mindaet al.; 2020; Párr. 18, 20-21)

\section{Rizotomía Facetaria}

Al no conseguirse un eficaz resultado mediante terapias conservadoras en base a fármacos, fisioterapias, reposo relativo, por ejemplo, o si persiste la intensidad de los síntomas, es cuando entonces se vuelve oportuno efectuar procedimientos especiales destinados a mitigar el dolor de este origen, tal es el caso de las neurotomías y los bloqueos facetarios.

Una Neurotomía Facetaria es la ablación o eliminación, por diversos métodos, de los pequeños nervios que transmiten la sensación de dolor exclusivamente desde las articulaciones facetarias de las vértebras afectadas. Así, en el lumbago, se dirigirá la neurotomía hacia las facetas de las vértebras lumbares, en general las 3 o 4 últimas con sus respectivas articulaciones. Este procedimiento es llevado a cabo con visión de rayos $X$, con el paciente despierto en la gran mayoría de los casos y con anestesia local. Es un procedimiento ambulatorio y con mínimas incomodidades, ya que se realiza a través de pequeñas punturas en los sitios afectados, sin incisiones.

La técnica más difundida y probada de realizar las neurotomías es a través de la radiofrecuencia. Con este método, una vez que el médico ha posicionado el electrodo en la articulación indicada guiado por rayos $X$ en el pabellón, se realiza una estimulación y luego una lesión de los nervios implicados en el dolor con radiofrecuencia, y el calor secundario a ella. La intervención completa dura alrededor de 30-40 minutos, es realizada en pabellón con anestesia local y en modalidad de paciente ambulatorio la gran mayoría de las veces. Además de la neuroto- mía, se realiza en el mismo acto un Bloqueo Facetario con anestésico y corticoides, que consiste en realizar el mismo procedimiento antes indicado, con la diferencia que no se aplica radiofrecuencia sino una solución de anestésico y corticoides para disminuir el dolor temporalmente. (Jarufe, s.f.)

Por su parte, Meli (2016) quien hace referencia al procedimiento con el término "Rizólisis", explica que se trata de "una técnica que permite tratar un tipo concreto de dolor de columna eliminándolo durante un periodo considerable de tiempo, incluso durante varios años. Se realiza mediante una lesión por calor de los nervios que transmiten este tipo de dolor". Adicionalmente detalla que en dicho procedimiento:

1. Se colocan con extrema precisión unos electrodos en forma de cánula capaces de producir la lesión térmica (se emplea una técnica de imagen en tiempo real que permite al médico posicionar estas cánulas exactamente en el objetivo terapéutico: la zona vertebral donde pasa el ramo medial del nervio espinal).

2. Alcanzado el punto diana, se acometen unas pruebas de seguridad por Rayos $X$ y por técnicas de neuroestimulación.

3. Una vez los chequeos han dado resultado positivo se realiza la lesión por termocoagulación, gracias a un generador muy sofisticado que proporciona energía de forma constante y controlada, a la temperatura y tiempo programados.

La rizólisis es el tratamiento de elección para el dolor de origen de las facetas articulares, es altamente efectivo y duradero. Tras su realización se logrará un buen control del dolor lumbar, cervical o torácico. Es fundamental que la rizólisis esté bien indicada, porque no es efectiva sobre cualquier dolor de columna. Es también indispensable que se lleve a cabo con la técnica y el equipamiento necesarios, para que funcione y dure en el tiempo. 
Estos fracasos del tratamiento son debidos a dos causas distintas: la selección del paciente y la técnica usada.

Si la rizólisis no quita el dolor es debido principalmente a que no estaba indicada, la rizólisis no es la panacea del dolor de espalda, sólo funciona en caso de dolor facetario.

Cuando, en cambio, sí ha funcionado pero su duración es inferior al año, el mal resultado está muy probablemente relacionado con la dimensión de la lesión. Debido a que los ramos mediales (nervios diana de la rizólisis) vuelven a crecer, si la lesión es pequeña, tardarán poco tiempo en regenerarse. (Meli, 2016)

Un aspecto que es igualmente importante mencionar es el relativo a los riesgos de esta, y en este sentido un equipo de especialistas mexicanos de la Unidad de Dolor y Cuidados Paliativos (2018), guiados por el Dr. Hugo Martínez, quien cuenta con más de 25 años de trayectoria médica y en la especialidad. aseguran que:

Realmente sus riesgos más graves son afortunadamente excepcionales y tienen que ver con la difusión excesiva del anestésico local, una lesión accidental de un nervio de la vecindad, o más raro aún, con la infección o el sangrado de la zona tratada. (Párr. 20)

Según Madariaga (2020) simplemente consiste en una "denervación" por radiofrecuencia (rizólisis por radiofrecuencia) de las terminaciones nerviosas que inervan las articulaciones facetarias de las vértebras en la espalda. Describe que con esta se trata de "romper" el circuito de señales dolorosas que tienen su origen en facetas bloqueando los nervios que se sitúan junto a ellas. Entre otros criterios expresados por la experta están los relacionados con las condiciones para su aplicación, ya que dice que procede sólo cuando haya sospecha probable de que la causa del dolor se deba a los nervios próximos a las articulaciones facetarias; y dejando ver que la exploración clínica y el uso de pruebas de imagen podrían bastar para sostener un diagnóstico correcto. Adicionalmente coincide con el criterio de otros especialistas al considerar que este tratamiento es pertinente cuando no se han alcanzado los resultados que esperados con otros tratamientos conservadores y las molestias sean de larga duración, intensase te impidan llevar una vida normal. En el mismo orden de ideas expuso que la rizólisis se aplica:

- Regularmente, para calmar el dolor si está bien localizado y no se irradia (se extiende) a otras zonas.

- Mientras tanto la persona no sienta pérdida de fuerza, sensibilidad o reflejos en brazos o piernas, pues de lo contrario, el origen del dolor pudiera ser distinto al de facetas articulares.

"Está demostrado que la aplicación de radiofrecuencia e infiltración sobre las terminaciones sensitivas que inervan dichas articulaciones consigue la remisión de la lumbalgia con alta tasa de éxito." (Instituto Aragonés de Traumatología, s.f.)

En este mismo sentido, como ejemplo, puede referirse los resultados que un equipo especialista, llevando a cabo un breve y práctico estudio, de hecho, han consiguieron hace ya un considerable tiempo con dicha terapia datos significativos. Tal es el caso del estudio de Mena, Achi, Riveros, \& Quintana (2008), quienes teniendo como objetivo la evaluación de la efectividad de la rizotomía facetaria en el manejo del dolor lumbar mecánico en una serie prospectiva y consecutiva de 31 pacientes, concluyeron de sus resultados que la misma pudo concebirse como una técnica efectiva, debido a que base a raíz de su aplicación lograron identificar una reducción significativa en el uso de analgésicos y por ende de la intensidad del dolor en los individuos sujetos al examen.

Otro trabajo que puede ser citado es el de

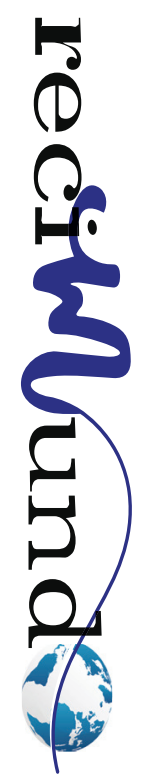


Aliaga et al. (2012), ya que enfocan su investigación, primordialmente, en la definición de la eficacia de la rizotomía del ramo medial con Radiofrecuencia en pacientes diagnosticados de síndrome facetario lumbar, y adicionalmente, en el establecimiento de la importancia del bloqueo como diagnóstico y criterio de selección de pacientes candidatos a rizotomía de ramo medial por RF. Con los resultados obtenidos logran determinar que:

Todos los pacientes mejoraron su condición en relación a antes de la Rizotomía con RF, el tiempo de mejoría total fue variable con un seguimiento de 3 años siendo la recurrencia del dolor en 2 pacientes a los 6 meses; 3 pacientes a los 10 meses y 2 pacientes a los 18 meses. [...] se encontró que los bloqueos facetarios fueron eficaces para el diagnóstico de

síndrome facetario lumbar en el 100\% de los pacientes (...). Estos hallazgos son consistentes con los resultados de varios estudios, como el realizado por Manchikanti et al. titulado «Lumbar facet joint nerve blocks in managing chronic facet joint pain: oneyear followup of a randomized, double-blind controlled trial: Clinical Trial NCT00355914», donde se encontró que el $82 \%$ de los pacientes mejoraron en cuanto al dolor, y el $78 \%$ de los pacientes mejoraron funcionalmente. Igualmente, en otro estudio realizado por el mismo investigador titulado «Evaluation of lumbar facet joint nerve blocks in managing chronic low back pain: a randomized, double-blind, controlled trial with a 2-year follow-up», se encontró que hasta el 90\% de los pacientes mejoraron sus síntomas de dolor un 50\% o más y en funcionalidad un 40\% o más. Adicionalmente, el estudio «Therapeutic efficacy of facet joint blocks» dirigido por Gorbach et al., con menos cantidad de pacientes, mostró que 31 de ellos (74\%) mejoraron de la sintomatología inmediatamente después del bloqueo y 14 pacientes (33\%) mejoraron a mediano plazo. (Pág. 18)
Un trabajo más reciente en el que específicamente se evalúa la efectividad analgesica de la rizotomia para el tratamiento del sindrome facetario lumbar, es el referido trabajo de Minda et al. (2020), quienes concluyeron que:

La rizotomía es un procedimiento eficaz para reducir el dolor, demostrando diferencias en el promedio de la escala EVA antes y después de la rizotomía lumbar. La rizotomía lumbar controlada es un método confiable para el manejo del dolor lumbar crónico en pacientes con síndrome facetario.

La Escala de Oswestry demuestra mayores valores antes de la rizotomía en comparación a los promedios después del procedimiento, lo cual refleja una disminución importante del dolor que se comprueba con una mejor capacidad funcional de la persona. La escala visual analógica (EVA), la escala de discapacidad de Oswestry y la escala EUROquol5-D demostraron ser eficaces instrumentos para medir el grado de dolor, discapacidad y calidad de vida de los pacientes antes y después de un procedimiento terapéutico.

Es importante utilizar la rizotomía lumbar como tratamiento principal del síndrome facetario lumbar por demostrase que es un método seguro, sencillo y eficaz para reducir la sintomatología de los pacientes. Además, siempre es aconsejable evaluar los resultados de procedimientos mínimos invasivos de la columna con el empleo de la Escala Visual Analógica para medir el grado de dolor de los pacientes con síndrome facetario antes y después del procedimiento terapéutico y la aplicación de la Escala de Oswestry para medir el grado de limitación funcional o discapacidad.

\section{Conclusiones}

De conformidad a la literatura encontrada puede inferirse que, en la actualidad, existen fundados estudios que, en relación al tratamiento del lumbago (lumbalgia o síndrome facetario lumbar) mediante la téc- 
nica de la rizotomía (rizólisis/denervación/ ablación por radiofrecuencia, diatermocoagulación o coagulación térmica) es el tratamiento de elección, ubicándose por encima de otros tipos de intervenciones mini-invasivas como es el caso de la infiltración de esteroides intra-articular (o facetario) u otros.

Dando por hecho que este tratamiento debe ser ejecutado sólo por el especialista con suficiente destreza y experiencia, es trascendental comprender que el éxito de esta técnica se apoya fundamentalmente en su ejecución con técnicas paralelas de TC y ecografías (sin menos cabo de la radioscopia); mismas que, por cierto, son igualmente útiles en el bloqueo nervioso (proceso previo, ineludible y clave de diagnóstico para la aplicación de la terapia neuroablativa); y es porque éstas favorecen una mayor exactitud en la punción.

\section{Bibliografía}

Aguilera, A., \& Herrera, A. (2013). Lumbalgia: Una dolencia muy popular y a la vez desconocida. Comunidad y Salud, 11(2), 80-89. Recuperado el 29 de 02 de 2020, de http://www.redalyc.org/articulo. oa?id=375740253010

AHRQ. (23 de Agosto de 2017). Consumer Summary: Agency for Healthcare Research and Quality. Recuperado el 29 de 02 de 2020, de Agency for Healthcare Research and Quality: https://effectivehealthcare.ahrq.gov/products/back-pain-treatment/espanol

Aliaga, M., Soliz, S., Molina, M., Jiménez, M., Ramos, V., Fernández, J., \& Ayala, C. (2012). Rizotomía de Facetas con Radiofrecuencia. Revista Boliviana del Dolor, 4(2), 13-19. Recuperado el 29 de 02 de 2020, de https://kipdf.com/download/ editorial-9-dr-ramiro-alvarado-articulo-original-rizotomia-de-facetas-dr-martin-_5b0451bf8ead0ec9208b458a.html

Enciclopedia llustrada de Salud de A.D.A.M. (20 de Marzo de 2018). Enciclopedia Médica: MedlinePlus. Recuperado el 29 de 02 de 2020, de MedlinePlus: https://medlineplus.gov/spanish/ency/ article/007425.htm

Enciclopedia llustrada de Salud de A.D.A.M. (13 de Mayo de 2019). Enciclopedia Médica: MedlinePlus. Recuperado el 29 de 02 de 2020, de MedlinePlus: https://medlineplus.gov/spanish/ency/ article/007422.htm
Fundación Española de Reumatología. (s.f.). Enfermedades: Fundación Española de Reumatología. Recuperado el 28 de 02 de 2020, de Sitio Web: Fundación Española de Reumatología: https:// inforeuma.com/enfermedades-reumaticas/lumbago-y-ciatica/

Instituto Aragonés de Traumatología. (s.f.). Tratamientos: Instituto Aragonés de Traumatología. Recuperado el 29 de 02 de 2020, de Sitio Web de: Instituto Aragonés de Traumatología: https:// aragontraumatologia.es/tratamiento-no-invasivo-del-dolor-de-espalda

Jarufe, F. (s.f.). Columna y Médula: Neuromed. (F. Jarufe, Editor) Recuperado el 28 de 02 de 2020, de Sitio Web de: Neuromed: http://www.neuromed.cl/articulo-bloqueos-y-neurotomias-en-dolores-de-columna.php

Madariaga, M. (14 de Enero de 2020). Tratamientos del dolor: Tuvidasindolor. Recuperado el 28 de 02 de 2020, de Sitio Web Tuvidasindolor: https://www. tuvidasindolor.es/rizolisis-lumbar-cervical-dorsal-radiofrecuencia/

Martínez, A., García, J., Ruiz, F., Guzmán, L., \& Castellano, M. (2017). Abordaje intervencionista del síndrome facetario lumbar: Denervación con radiofrecuencia. Revista Chilena de Radiología, 23(1), 7-14. Obtenido de La rizolisis de la rama medial del ramo dorsal

Meli, M. (15 de Diciembre de 2016). Rizólisis, la clave de su eficacia: Aliviam. Recuperado el 28 de 02 de 2020, de Sitio Web de Aliviam: http://www.aliviam. com/es/blog/2016/12/15/rizolisis-dolor-facetario/

Mena, I., Achi, J., Riveros, R., \& Quintana, L. (2008). Efectividad de la rizotomía facetaria en el manejo del dolor lumbar mecánico. Revista Chilena de Neurocirugía, 31(2), 11-13. Recuperado el 29 de 02 de 2020, de https://imbiomed.com.mx/1/1/articulos. php?method=showDetail\&id_articulo $=55128 \& i d \_$ seccion=2646\&id_ejemplar $=5581 \&$ id_revista $=118$

Minda, J., Saavedra, M., García, C., \& López, K. (2020). Efectividad analgesica de la rizotomia para el tratamiento del sindrome facetario lumbar. Sinergias educativas, E(1). Recuperado el 29 de 02 de 2020, de http://www.redalyc.org/articulo. oa?id=573561701020

NINDS. (21 de Diciembre de 2016). Dolor Lumbar: NINDS. Recuperado el 29 de 02 de 2020, de Sitio Web de: National Institute of Neurological Disorders and Stroke: https://espanol.ninds.nih.gov/ trastornos/dolor_lumbar.htm\#wrapper

Rodríguez, A. (07 de Febrero de 2020). Spine Universe. Recuperado el 29 de 02 de 2020, de Sitio 
web de: Spine Universe: https://www.spineuniverse.com/espanol/dolor-espalda/sindrome-articulacion-facetaria

Unidad de Dolor y Cuidados Paliativos. (14 de Noviembre de 2018). Inicio: Unidad de Dolor y Cuidados Paliativos. Obtenido de Sitio Web de: Unidad de Dolor y Cuidados Paliativos: https://unidaddedolor.wordpress.com/2018/11/14/que-es-la-rizotomia/

\section{CitAR ESTE ARTICULO:}

Pillco Guaman, E., Pañega Narváez, M., López Aldas, R., \& Cusco Rivera, C. (2020). Lumbago: rizotomia facetaria. RECIMUNDO, 4(1(Esp)), 143-154. doi:10.26820/recimundo/4.(1).esp.marzo.2020.143-154

(c) $(1)(9)$

RECONOCIMIENTO-NOCOMERCIAL-COMPARTIRIGUAL CC BY-NC-SA

ESTA LICENCIA PERMITE A OTROS ENTREMEZCLAR, AJUSTARY CONSTRUIR A PARTIR DE SU OBRA CON FINES NO COMERCIALES, SIEMPRE Y CUANDO LE RECONOZCAN LA AUTORÍA Y SUS NUEVAS CREACIONES ESTÉN BAJO UNA LICENCIA CON LOS MISMOS TÉRMINOS. 